\title{
Biopsy Techniques for Eyelid Tumours
}

\author{
Albert Wu, Shyamala C. Huilgol, and Dinesh Selva
}

\section{Introduction}

Biopsies of eyelid lesions can be performed to establish the diagnosis and may identify high-risk histological tumour subtypes. This may influence management, especially when there is a significant cosmetic impact of excising a large lesion, when non-surgical management can be used or when planning Mohs surgery. Another use is establishing tumour boundaries through mapping biopsies. A biopsy may also assist in determining whether tumour recurrence has occurred following previous treatment [1]. The main types of biopsy are excisional, incisional, punch and shave biopsies. A biopsy may be performed with the intention of excising a lesion, for example, in the case of a very small basal cell carcinoma (BCC) where the clinician feels confident about the diagnosis. It should be borne in mind that biopsies can occasionally provide an inaccurate or incomplete diagnosis due to sampling errors.

Electronic supplementary material The online version of this chapter (https://doi.org/10.1007/978-3-030-18757$6 \_2$ ) contains supplementary material, which is available to authorized users.

A. Wu $(\bowtie) \cdot$ S. C. Huilgol

Dermatology Department, Royal Adelaide Hospital, Adelaide, SA, Australia

D. Selva

Ophthalmology Department, Royal Adelaide

Hospital, Adelaide, SA, Australia
In one study of periocular BCC with aggressive subtypes $(n=51)$, biopsy failed to identify an aggressive component in $52 \%$ of cases [2].

\section{Preoperative Considerations}

Although biopsies are generally straightforward procedures, the patient's medical history should first be reviewed $[1,3]$. General medical history, allergies and medications should be noted. Smoking and medical conditions may impair healing. Antiplatelets, anticoagulants, bleeding disorders and hypertension increase the risk of haemorrhage. Informed consent should be obtained and complications explained such as haemorrhage, infection, scarring, ectropion and pain. The lesion may be outlined with a skin marker pen before the procedure to help identify the lesion following injection of local anaesthesia [1, $3]$. A photo with the biopsy site marked and measurements to fixed landmarks such as the canthi or lacrimal puncta are essential for later accurate localisation, especially for smaller lesions which may be very difficult to find after the biopsy.

\section{Biopsy Techniques}

The main techniques utilised in the periocular area are excisional, incisional, punch, snip, curette and shave biopsies. Techniques are selected based on lesion characteristics, possible diagnosis, loca- 
tion and surgeon preference. For instance, a snip biopsy may be used for a pedunculated lesion, or a shave biopsy for an elevated lid margin lesion. One study of periocular tumours $(n=20)$ found that incisional biopsy had an accuracy rate of $95 \%$ [4]. Similarly, the accuracy rate of punch biopsies in identifying periocular tumours has been reported to be $67-85 \%$ [4-6]. Shave biopsy may produce a better cosmetic outcome than a deeper biopsy which may leave a notch in the eyelid if the lesion proves to be benign. However, shave biopsy is not recommended in certain scenarios such as differentiating keratoacanthoma-type squamous cell carcinoma (SCC) from invasive SCC $[1,3]$ or in suspected melanoma where tumour thickness is an important prognostic factor.

\section{Equipment Required for Biopsy}

- Skin preparation materials

- Local anaesthetic with adrenaline 1:100,000

- Forceps

- Scalpel/scissors

- Cautery or other method of haemostasis

- Formalin container(s)

Other equipment can be added as needed, such as disposable punch, blade for shave biopsy and suturing equipment. An eye shield may be used to prevent eye injury.

\section{Incisional Biopsy Technique}

(Video 2.1)

1. Aseptic skin preparation.

2. Inject local anaesthetic with adrenaline around the lesion.

3. Using a scalpel blade (No. 11 blade often useful for small lesions), aim to sample a piece of tumour and some adjacent normal tissue. Avoid sampling ulcerated areas which may only provide necrotic tissue and not provide a diagnosis. Also avoid keratinised/crusted areas and remove overlying crust with irrigation/soaking if necessary. An elliptical excision can also be performed.
4. Place specimen in formalin.

5. Cauterise wound or use pressure.

6. Close wound with sutures if necessary but small defects can be left to heal by secondary intention $[1,7,8]$.

\section{Punch Biopsy Technique (Video 2.2)}

1. Aseptic skin preparation.

2. Inject local anaesthetic with adrenaline around the lesion.

3. Using thumb and index finger, stretch the skin on either side of the lesion away from the lesion. This produces an oval defect which facilitates wound closure. Align the oval defect with skin tension lines.

4. Insert punch into lesion perpendicular to skin with a twisting motion. Aim to sample a noncentral part of the lesion away from the margin. Take care not to injure the globe. Consider using an eye shield.

5. Remove the punch perpendicular to skin, leaving behind the whole biopsy specimen.

6. Using forceps, grasp an edge of the specimen gently to avoid crush artefact, and lift the specimen out. The specimen may also be lifted out using a needle, a skin hook or a suture passed through the specimen. This step may be made easier by pushing down on surrounding skin to raise the lesion relative to the surrounding skin.

7. Cut the base of the specimen with scissors or scalpel.

8. Place specimen in formalin.

9. Consider cautery. Haemostasis may also be achieved with sutures and slight pressure. Suture closure produces a better cosmetic outcome but is not always necessary $[1,3,4,6,9]$.

\section{Shave Biopsy Technique (Video 2.3)}

1. Aseptic skin preparation.

2. Inject local anaesthetic with adrenaline intradermally, raising the lesion from the surrounding skin.

3. Stretch the skin. When a lesion has limited elevation, the skin may be bunched up to 
increase elevation. With raised lesions, forceps may be used to lift the lesion.

4. A scalpel is often used on the eyelid. The blade is used to saw through the lesion horizontally along the skin or the eyelid margin. Razor blades are used elsewhere on the body but are difficult to use on or near the eyelid margin.

5. Place specimen in formalin.

6. Apply light pressure, chemical haemostasis or cautery. Wound re-epithelialisation occurs in around 1 week $[1,3,7,8]$.

\section{Snip Biopsy}

Lift the lesion with forceps to snip the base with scissors. The lesion should not be pulled too far, resulting in removal of more skin at the base than necessary. Light pressure may be sufficient for haemostasis [1].

\section{Curette Biopsy}

Curette biopsy may be used for soft superficial lesions, including seborrhoeic keratosis, actinic keratosis and some BCC. Stretch the surrounding skin, and scoop down through the lesion with the curette. Avoid this method on lax skin of the eyelid as the curette may tear the skin while attempting to remove the tumour. Light pressure, chemical haemostasis or electrodessication can be used [1].

\section{Communication with Pathologist}

The pathology request form and specimen container should be labelled with patient identifying details, date and biopsy site. Ensure the specimen is in the container. The request form should also include details of the requesting clinician, type of biopsy (diagnostic or excisional), and relevant clinical information such as suspected clinical diagnosis and previous treatments to the biopsy site. A diagram or clinical photograph may be included. Specific questions may be included on the request form, and the case may be discussed with the pathologist if required [1]. The clinician should record all specimens sent to the pathologist, and follow up and act upon pathology results in a timely manner.

\section{Postoperative Care}

Vaseline may be applied to the wound site. Topical antibiotics should not be routinely used. The site should be cleaned regularly, and medical advice should be sought if signs of infection develop.

\section{Conclusion}

Biopsy of the eyelid is an important skill which assists in diagnosis and management of eyelid tumours. The main techniques are excisional, incisional, punch and shave biopsies, and are selected depending on the clinician's preference and characteristics of the lesion.

\section{References}

1. Robinson JK, Hanke CW, Siegel DM, Fratila A, Bhatia A, Rohrer TE. Surgery of the skin: procedural dermatology. 3rd ed. London/New York: Elsevier/Saunders; 2015.

2. Sun MT, Wu A, Huilgol SC, Selva D. Accuracy of biopsy in subtyping periocular basal cell carcinoma. Ophthal Plast Reconstr Surg. 2015;31:449-51.

3. Wheeland RG. Cutaneous surgery. Philadelphia: W.B. Saunders; 1994.

4. Rice JC, Zaragoza P, Waheed K, Schofield J, Jones CA. Efficacy of incisional vs. punch biopsy in the histological diagnosis of periocular skin tumours. Eye (Lond). 2003;17:478-81.

5. Chatterjee S, Moore S, Kumar B. Punch biopsy in the management of periocular basal cell carcinomas. Orbit. 2004;23:87-92.

6. Carneiro RC, de Macedo EM, de Lima PP, Bonatti $\mathrm{R}$, Matayoshi S. Is 2-mm punch biopsy useful in the diagnosis of malignant eyelid tumours? Ophthal Plast Reconstr Surg. 2012;28:282-5.

7. Dutton JJ, Gayre GS, Proia AD. Diagnostic atlas of common eyelid diseases. New York: Informa Healthcare; 2007.

8. Biswas A. Eyelid tumours: clinical evaluation and reconstruction techniques. New Delhi: Springer; 2014.

9. Sassani JW. Punch biopsy technique for the ophthalmologist. Arch Ophthalmol. 1991;109:464-5. 
Open Access This chapter is licensed under the terms of the Creative Commons Attribution 4.0 International License (http://creativecommons.org/licenses/by/4.0/), which permits use, sharing, adaptation, distribution and reproduction in any medium or format, as long as you give appropriate credit to the original author(s) and the source, provide a link to the Creative Commons license and indicate if changes were made.

The images or other third party material in this chapter are included in the chapter's Creative Commons license, unless indicated otherwise in a credit line to the material. If material is not included in the chapter's Creative Commons license and your intended use is not permitted by statutory regulation or exceeds the permitted use, you will need to obtain permission directly from the copyright holder.

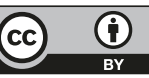

